\title{
LA PROMOCIÓN DE LA LECTURA EN LA ESCUELA: EL CASO DEL PLA D'IMPULS A LA LECTURA EN CATALUÑA
}

\section{THE PROMOTION OF READING IN SCHOOL: THE CASE OF PLA D'IMPULS TO READING IN CATALONIA}

\author{
Moisés Selfa Sastre* \\ Enric Falguera Garcia**
}

\begin{abstract}
Resumen: En este artículo, de carácter eminentemente teórico, presentamos qué es el Pla d'Impuls a la Lectura (Plan de Impulso a la Lectura) (PIL), una estrategia impulsada en las escuelas de Cataluña, desde el año 2011, para fomentar el hábito lector y el desarrollo de la competencia lectora en escolares de Educación Primaria (5-12 años) y Educación Secundaria (12-16 años). Después de definir los objetivos y ejes básicos de este PIL, abordamos la epistemología de este en relación a tres conceptos básicos: saber leer, leer en las áreas del currículum y el gusto por la lectura. Finalmente, nos referimos al papel de las Bibliotecas Escolares y las características de la Lectura Digital tal como las concibe el PIL.

Palabras clave: Lectura. Escuela. Impulso a la Lectura. Competencia Lectora. Lectura Digital.
\end{abstract}

\begin{abstract}
In this paper, eminently of theoretical nature, we present what is the Pla d'Impuls a la Lectura (Plan of Impulse to Reading) (PIL), a strategy promoted in the schools of Catalonia, since 2011, to encourage the reading habit and the development of the reading competence in Primary school students (5-12 years) and Secondary Education (12-16 years). After defining the objectives and basic axes of this PIL, we address the epistemology of this in relation to three basic concepts: knowing how to read, reading in the areas of curriculum and the taste for reading. Finally, we refer to the role of School Libraries and the characteristics of Digital Reading as they are conceived by the PIL.
\end{abstract}

Key-Words: Reading. School. Promoting Reading. Reading Competence. Digital Reading.

\section{El Pla d'Impuls a la Lectura: descripción}

En el acuerdo marco de carácter legislativo para luchar contra el fracaso escolar, aprobado por el Gobierno de Cataluña en fecha 8 de febrero de 2011 (GENCAT, 2013: 21), se ponen las bases para combatir el fracaso escolar, uno de los problemas más importantes en la educación y que se convierte en un objetivo prioritario de las políticas educativas del Gobierno.

El Plan de Gobierno 2011-2014, de 3 de mayo, identifica como reto de país la mejora de la educación y la formación a lo largo de la vida, y en el eje 2, relativo a la enseñanza, establece como objetivo reducir el fracaso escolar potenciando la lectura sistemática a lo largo de toda la educación básica (Educación Primaria y Educación secundaria obligatoria) mediante un plan nacional de lectura (Pla d'Impuls a la Lectura).

Es en este contexto que el Gobierno de Cataluña impulsa este Pla d'Impuls a la Lectura, que vertebrando los esfuerzos de diferentes sectores y ámbitos de la sociedad catalana, tiene como finalidad aumentar los hábitos lectores de los ciudadanos y su competencia lectora, ya que es la garantía de una plena participación de estos en la sociedad actual.

\footnotetext{
* Professor Agregat da Universitat de Lleida - Espanha. Didàctica de la Llengua i la Literatura. E-mail: mselfa@didesp.udl.cat

** Universitat de Lleida Espanha .Coordinador del Màster en Formació Avançada del Professorat d'Infantil i Primària. E-mail: efalguera@didesp.udl.ca
} 
El Departament d'Ensenyament (DE) -órgano administrativo del cual depende la escuela catalana- participa en este PIL estableciendo como objetivo estratégico la mejora del éxito escolar de todos los alumnos. Y lo hace potenciando la lectura sistemática en todas las áreas y materias del currículo a lo largo de toda la educación básica para aumentar la competencia comunicativa lingüística y el desarrollo del hábito lector de los niños y jóvenes, teniendo presente que la competencia lectora es la base de muchos de los aprendizajes y un requisito para participar con éxito en buena parte de los ámbitos de la vida adulta.

Para alcanzar este objetivo estratégico el DE facilita a los centros orientaciones, pautas, asesoramiento y formación para los profesores, los equipos directivos y las familias para que incorporen y sistematicen estrategias lectoras como eje vertebrador de los aprendizajes, cada uno desde su ámbito de responsabilidad (GENCAT, 2013).

\section{Objetivos y ejes básicos de actuación del PIL}

El PIL (GENCAT, 2011-2014) tiene como objetivos generales los siguientes: mejorar la competencia lectora para aumentar el rendimiento académico de todos los alumnos y favorecer así el éxito educativo; conseguir que los niños y jóvenes asuman sus responsabilidades como aprendices autónomos con capacidad para acceder a la información y al conocimiento, haciendo de la lectura la herramienta para el aprendizaje en todas las áreas y materias del currículo; y formar buenos lectores que disfruten y aprendan leyendo.

En este sentido, también se contemplan varios objetivos estratégicos para todos los sectores que conforman la comunidad educativa. Para los alumnos se prevé alcanzar los siguientes objetivos: incorporar la autoevaluación como estrategia de aprendizaje que las permita autorregular el proceso de comprensión; incrementar el tiempo de dedicación a la lectura y el número de libros leídos a lo largo del año; incorporar la reflexión sobre qué y cómo se ha aprendido, explicitando las estrategias de lectura que se utilizan; y elaborar un dosier de lectura que recoja los progresos en el fomento de esta competencia.

En cuanto a los profesores, los objetivos son los siguientes: planificar las unidades didácticas desarrollando las estrategias metodológicas de acuerdo con la evolución de los aprendizajes de sus alumnos; incorporar la evaluación como herramienta del mismo proceso de enseñanza; y articular los objetivos de las actividades a partir de lo que los alumnos deberán demostrar que han aprendido.

Para los centros educativos, se aspira a alcanzar los siguientes objetivos: incorporar la lectura y las estrategias de comprensión lectora en todas las áreas; y establecer una estrategia de centro adecuada a las necesidades diagnosticadas a partir de los resultados de evaluación interna, externa y complementada con las evaluaciones generales del sistema.

Para las familias, las actuaciones recomendadas van en la siguiente dirección: encontrar uno tiempo diario (de 10 a 20 minutos) para leer con los niños y leer a su lado para reforzar los hábitos de buen lector y la lectura autónoma; conocer la metodología del aprendizaje de la lectura que se trabaja desde la escuela; y aplicar las orientaciones de cómo pueden contribuir a hacer de sus hijos unos buenos lectoras.

Este PIL no está concebido únicamente en relación a unos objetivos a alcanzar, sino que también se fundamenta en el siguiente eje genérico de actuación: leer para aprender. 
Leer para aprender supone concebir el uso de la lectura para el aprendizaje. Los alumnos deben desarrollar su responsabilidad en el aprendizaje y tener la capacidad de utilizar la lectura como herramienta para el acceso a la información, el descubrimiento y la ampliación de conocimientos, es decir, el uso de la lectura como instrumento de aprendizaje en todas las áreas del currículo (ROSE; MARTÍN, 2018).

Del Leer para aprender se derivan dos ejes de actuación que desarrollaremos en las líneas posteriores cuando fundamentemos la epistemología del PIL: saber leer y el gusto por la lectura.

\section{Epistemología del PIL: saber leer, leer en las áreas, el gusto por leer.}

\section{Saber leer}

Saber leer significa situar el aprendizaje de la lectura como desarrollo de la capacidad de leer, comprender e interpretar textos (SOLÉ, 2012). El logro de las habilidades funcionales básicas de lectura corresponde al ciclo inicial de la educación primaria y es en este ciclo donde se deben tomar las medidas de apoyo necesarias con los alumnos que presentan dificultades. Corresponde a los ciclos posteriores continuar el trabajo de las estrategias lectoras, adaptadas a las necesidades de cada momento.

En el Marco teórico de las pruebas PISA (PISA, 2009), se habla de enseñanza de la competencia lectora (CL) y no de comprensión de un texto, porque se quiere focalizar la atención en los usos de los textos, reconociendo que, evidentemente, no se pueden usar adecuadamente si no se entienden. Esto quiere decir que, al enseñar a leer, es necesario que, además de las actividades de identificación de los signos y formatos, se propongan otras para construir e inferir el significado del texto. Esto, sin embargo, no es todo lo que hay hacer, ya que, para saber leer, hay que dominar también las reglas de uso de los textos en los contextos en que aparecen y se utilizan, y reconocer la función social que tiene cada uno, identificando las opiniones y las ideologías que transmite o están implícitas. Porque leer para aprender a pronunciar letras no crea necesariamente buenos lectores. Así, pues, el aprendizaje de la lectura no se centra solo en la comprensión lectora literal, sino que hace hincapié en las comprensiones inferencial y crítica.

La CL, por tanto, constituye una capacidad amplia, compleja y transversal, que se empieza a desarrollar incluso antes de lo que se conoce como proceso de enseñanza de la lectura. Cuando alguien cuenta un cuento a un niño, o le canta una canción y lo ayuda a interpretar lo que le está diciendo, ya se inicia el proceso de desarrollo de la CL (SOLÉ, 2013).

La CL es una capacidad compleja y transversal, determinada social y culturalmente. La comunicación en un sentido amplio, hecha con todo tipo de soportes y formatos, se ha convertido en un elemento clave en la sociedad actual. Todo el mundo necesita desarrollar la competencia comunicativa en general, y la lectora en particular, para devenir ciudadano con plenitud y responsabilidad. Con el desarrollo de las tecnologías de la información y la comunicación (TIC), aparecen nuevas situaciones letradas que plantean nuevos retos para los lectores, situaciones diversas y cambiantes en que se lee, en nuevos formatos y soportes, para aprender nuevos conocimientos, que ayuden a entender el mundo; a comprender las otras personas y a uno mismo; resolver problemas y encontrar alternativas a estos.

Desde este punto de vista, hay que aprender a usar la lectura como herramienta eficaz para actuar eficientemente y resolver las situaciones complejas y cambiantes en 
que los alumnos se puedan encontrar (SOLÉ, 2000). Es necesario que el alumnado se dé cuenta de la necesidad de hacer crecer y movilizar todos sus recursos para usar los textos. Por ello se deben considerar todas las posibles situaciones comunicativas letradas, desde las más cercanas a la realidad escolar, las que se crean en los centros educativos, tanto las más académicas como las que no lo son tanto, hasta las más alejadas, las que aporta la diversidad del alumnado del centro y las de su entorno más cercano.

Del mismo modo, la CL supone las capacidades de actuar para afrontar situaciones reales, cambiantes y diversas, de entender el mundo y de atender objetivos específicos que permitan identificar y resolver eficazmente las situaciones cotidianas.

Para leer de una manera eficaz y reflexiva, habrá que saber movilizar los recursos pertinentes, centrando el aprendizaje en los procesos cognitivos de la lectura, en el cómo se lee y qué mecanismos participan del proceso lector. Por ello, la enseñanza competencial debe atender prioritariamente la forma en que se adquieren estos recursos y cómo se integran en aprendizajes más globales. De hecho, las competencias progresan según el dominio que se tiene de estos recursos personales, al igual que su mayor desarrollo mejorará el dominio de la lectura (SELFA; VILLANUEVA, 2015). Las actitudes positivas son fundamentales para el progreso de los hábitos lectores. Hay una actitud personal de implicación en el proceso lector, considerando la lectura como herramienta capital para convertirse en miembros activos de la sociedad. Esto implica que el lector se sienta motivado y tenga interés por la lectura, para desarrollar su autonomía, su capacidad de tomar decisiones y de regular el proceso para conseguir los objetivos que pretende. En todo este proceso de aprendizaje es primordial la interacción con los textos y con otros lectores para conseguir la mejora de las capacidades.

\section{Leer en las áreas curriculares}

Con pocas diferencias, los currículos de Primaria y de Secundaria en Cataluña remarcan que la competencia en comunicación lingüística es la herramienta básica para vehicular todos los aprendizajes y, por tanto, su desarrollo es responsabilidad de todas las áreas y materias del currículo.

En cuanto a la lectura, el criterio también es claro. El Decret 142/2007 d'Ordenació de l'Educació Primària afirma que la lectura es un factor fundamental para el desarrollo de las competencias básicas y debe estar incluida en el desarrollo de todas las áreas.

El enfoque competencial del currículo pide que los equipos docentes velen por la integración de los conocimientos, la funcionalidad de los aprendizajes y la autonomía personal del alumnado, es decir, el aprendizaje significativo. Con este planteamiento, el trabajo de comprensión lectora pierde su sentido si no tiene un objetivo claro integrado dentro de una tarea. Las áreas no linguísticas constituyen, por tanto, excelentes contextos de uso real de la lectura. Esta responsabilidad compartida se justifica por el papel esencial que juega la lectura en la construcción de conocimiento (CLAR; LLAURADÓ, RIERA, QUINQUER; ROCA, 2007). A fecha de hoy difícilmente podríamos encontrar ningún docente que discute la importancia del hecho lector como clave de acceso a los contenidos.

En la secuencia didáctica el texto a menudo es el elemento central de trabajo, a partir del cual se pueden revisar determinados aprendizajes, generar otros nuevos o confrontar hipótesis en el aula. No solo por su papel primordial de transmisor de información, sino porque hace posible la negociación de significados que debe permitir 
que el alumnado construya conocimiento de manera personal y colectiva a partir de lo que ya sabe permite pensar y aprender (SOLÉ, 2011), dicho de otra forma, a partir de sus conocimientos previos.

El contenido de las diferentes áreas se suele presentar en textos expositivos, con mucha densidad de conceptos y un lenguaje marcadamente abstracto y metafórico, lo que se denomina lenguaje académico. Es un lenguaje altamente especializado, porque cada disciplina tiene un patrón lingüístico que le es propio, coherente con su temática, que se caracteriza por la especificidad del léxico, las construcciones gramaticales y las estructuras textuales empleadas. Esto hace que, si las habilidades lectoras solo se trabajan en las áreas de Lengua, la transferencia de estas habilidades en la comprensión de un texto de las otras áreas no siempre será fácil ni inmediata, porque es necesario tener en cuenta este patrón lingüístico para acceder a la información del texto (LOMAS, 2003). Aprender a leer en las áreas no lingüísticas es, por tanto, aprender a acceder a los contenidos del área, y las actividades de comprensión de textos didácticos son actividades de aprendizaje. Hay que tener claro, sin embargo, que no se trata de que todos los docentes se conviertan en profesores de Lengua, sino que trabajen aquellos aspectos del lenguaje académico necesarios para garantizar el éxito dentro de su área.

Hay que trabajar desde todas las áreas la estructura de las tipologías textuales en diversidad de textos de medios diferentes, enmarcados en situaciones comunicativas reales. También es importante proponer actividades diferentes y plantearlas de manera variada, para activar unas habilidades u otras en cada caso.

Las pruebas PISA nos confirman que hay que ir por este camino cuando nos hablan de textos continuos, discontinuos y multimodales, y formulan una definición sobre la competencia lectora que implica la comprensión del texto, pero también la reflexión y la implicación, con varios objetivos, uno de los cuales es el siguiente: la competencia lectora consiste en comprender y utilizar los textos escritos y reflexionar y implicarse para alcanzar los objetivos propios, desarrollar el conocimiento y el potencial de cada uno y participar en la sociedad (MARCO PISA, 2009).

\section{Gusto por leer}

Por último, el gusto por leer se adquiere desarrollando el hábito lector $\mathrm{y}$ fomentando la lectura sistemática en cualquier soporte, aprendiendo a reflexionar sobre lo leído, sabiéndolo explicar mediante evidencias orales o escritas y sabiéndolo compartir

La conexión con la experiencia vital y el disfrute de los textos han pasado a considerarse un elemento clave para el acceso a la lectura y la consecución de hábitos permanentes. Desde hace décadas la escuela ha hecho un gran esfuerzo para acercarse a los intereses de los alumnos de medios sociales y de hábitos culturales muy diversos con el fin de impulsarlos hacia la lectura personal. Este apartado del plan de lectura trata justamente de cómo conseguir esta conexión vital entre la lectura y los discentes. La extensión de las prácticas de animación lectora y los debates sobre la lectura libre y la lectura obligatoria han hecho verter ríos de tinta sobre su conveniencia y las condiciones de su éxito en la formación lectora. En este periodo, las administraciones educativas de todos los países de nuestro entorno han intentado regular la lectura escolar combinando varios criterios didácticos en relación tanto a la lectura prescriptiva como un espacio escolar de lectura autónoma (DURAN; MANRESA, 2008). Asimismo, el esfuerzo para el fomento de la lectura por placer ha traspasado el ámbito escolar y la mayoría de países han puesto en marcha múltiples campañas y planes de lectura de alcance, tipo de 
actividades y resultados muy diversos (SÁEZ; CLARIANA; CLADELLAS; BADIA; GOTZENS, 2014). Las administraciones catalanas hace años que impulsan iniciativas de fomento de la lectura. La administración educativa inició en 1986 el programa denominado precisamente El gust per la lectura con el objetivo de conseguir que los niños y jóvenes se conviertan lectores competentes, críticos y capaces de disfrutar del placer de la lectura. Desde entonces, el programa ofrece formación para la dinamización de la lectura, material didáctico (como guías de las obras), actividades complementarias (rutas literarias, cine fórum, visitas guiadas, jornadas, debates), etc.

\section{Actuaciones a desarrollar para dar cumplimiento al desarrollo del PIL}

Para poder dar cumplimiento a este PIL se han previsto un conjunto de actuaciones, entre las que cabe destacar las siguientes.

En relación al sistema educativo, el DE impulsa la competencia lectora como un elemento fundamental en la práctica diaria de los docentes y de los equipos directivos, la formación inicial de los docentes (Grados de Maestro y Másters de Educación), la revisión de los planes y proyectos de centro y de entorno, el sistema de acceso a la función docente y la formación y el sistema de acceso a la dirección

En cuanto a los centros educativos, cada curso se seleccionará 8 centros por área geográfica de Inspección (4 de primaria y 4 de secundaria), que dispondrán de una formación específica combinando las modalidades de seminario y asesoramiento. Serán centros con prácticas exitosas de lectura, que compartirán con otros centros, y centros que aplicarán estrategias para la mejora de la competencia lectora y la incentivación de la lectura.

Con carácter general, se llevarán a cabo conferencias, talleres de lengua y literatura, el congreso de Educación y Entorno: Lectura, familia y entorno, itinerarios literarios y jornadas de intercambios de experiencias.

También hay que destacar los programas de apoyo al impulso de la lectura, como son El gust per la lectura, el Pla d'Actualització del Programa d'Immersió Lingüistica y el Programa de Biblioteques Escolars Puntedu.

\section{La Biblioteca Escolar y el desarrollo de los hábitos de lectura literaria}

La Biblioteca Escolar aparece como uno de los factores clave en el proceso de acceso y consolidación del hábito lector. Los estudios sobre los resultados de la evaluación PISA, por ejemplo, remarcan que los alumnos de las escuelas con bibliotecas escolares activas obtienen mejores resultados. En este campo, queda mucho por hacer en nuestro marco educativo. El estudio de MARCHESI y MIRET (2005: 57), sobre las escuelas de todo el estado español, revela que las bibliotecas escolares en España ocupan los últimos lugares de los países europeos en casi todos los aspectos (existencia, fondos, acceso a la red, presupuesto, personal encargado, etc.) y que el $40 \%$ del profesorado no las utiliza en todo el curso. La falta de su uso es aún más acentuada en la etapa Educación Secundaria: un $75 \%$ del profesorado no va nunca con sus alumnos, mientras que, del alumnado, solo la utiliza el 50\% (cuando en Educación Primaria lo hace el 70\%). Aunque no tenemos datos más recientes sobre las bibliotecas escolares catalanas, hay que decir que estos últimos años, concretamente desde el curso 2005-2006, se ha iniciado el proyecto llamado Puntedu con el objetivo de que en cada 
centro educativo haya un espacio de conocimiento y aprendizaje, que trabaje con el objetivo de impulsar y consolidar el funcionamiento de la biblioteca, entendido como centro de recursos para el aprendizaje y la investigación y como un servicio para el desarrollo del currículo y del proyecto educativo del centro.

Así, la adquisición de hábitos de lectura va muy ligada a la lectura literaria, especialmente a la lectura de cuentos y narraciones, a la que los niños y adolescentes dedican mayor tiempo y esfuerzo con el objetivo de dominar el lenguaje y el escrito (SELFA; BALÇA; COSTA, 2017). Este es un hecho aún más relevante si recordamos que los estudios de las evaluaciones PISA señalan la lectura de textos largos como uno de los factores más influyentes en los buenos resultados de comprensión lectora. La capacidad formativa de la literatura ha sido expuesta múltiples veces (CERRILLO, 2010). Solo recordaremos aquí, pues, que con el lenguaje se da sentido a la realidad y que la literatura justamente explora las posibilidades del lenguaje en múltiples ámbitos del saber y la experiencia. La literatura, además, ofrece unas cualidades formativas para el individuo -estéticas, cognitivas, afectivas y linguiísticas- que contribuyen a la formación de la persona, una formación que va indisolublemente ligada a la construcción de la sociabilidad y que se realiza a través de la confrontación con textos que explicitan la forma en la que las generaciones anteriores y las contemporáneas han abordado y abordan la valoración de la actividad humana. A través de la educación literaria en la escuela, los chicos y las chicas aprenden muchos de los referentes de su cultura, perciben las jerarquías en el funcionamiento social de los textos, obtienen recursos para apreciar los niveles de significado que ofrecen las obras, adquieren una manera de hablar sobre los libros, se les abre la posibilidad de objetivar la lectura según varios criterios culturales, como el de género, etapa histórica, etc., y pueden interrelacionar las competencias adquiridas con otros sistemas artísticos como los dramáticos y los audiovisuales.

La educación literaria se entiende como el desarrollo de la competencia en la interpretación de los textos (BALLESTER, 2015). Esto supone la necesidad de prever dos líneas de fuerza paralelas a lo largo de todas las etapas educativas para que se produzca el aprendizaje: la adhesión afectiva a través de la percepción de uno mismo como perteneciente a esta comunidad interpretativa y el aprendizaje de las convenciones que rigen las formas literarias de manera que se pueda construir el máximo de sentido. Además existe la necesidad de una práctica educativa que se desarrolla a través de la recepción oral o la lectura directa de los textos por parte de los aprendices, por un lado, y las formas guiadas para enseñar la manera de construir sentidos cada vez más complejos, por otro.

\section{La lectura digital y el PIL}

No se lee de la misma manera según el soporte, impreso o digital, en papel o en pantalla. Hay afinidades y diferencias considerables que vienen dadas, fundamentalmente, por la forma en que se estructuran y entrelazan los textos, lo que condiciona la demanda cognitiva de quien lee. Mientras el texto impreso es un texto fijo con unos límites definidos, el digital queda marcado por la calidad de hipertexto. Se trata de un texto o textos con herramientas y características de navegación que hacen posible, e incluso requieren, una lectura no secuencial. Cada lector se construye un texto a medida a partir de la información que encuentra en los enlaces que sigue (CORDÓN, 2016). 
En el soporte digital, generalmente solo se ve una pequeña parte del texto ya que a menudo se desconoce la extensión total del texto disponible. Las herramientas y características de navegación tienen un papel muy importante en el soporte digital. Los lectores competentes de textos digitales deben estar familiarizados con el uso de estos dispositivos. Además, una buena parte de actividades de lectura digital implican el uso de una gran cantidad de textos, a veces elegidos a partir de un conjunto prácticamente infinito. Los lectores deben estar familiarizados con las herramientas de recuperación, indexación y navegación por los enlaces entre los textos. El menú y el enlace son las herramientas de navegación más comunes que permiten generar documentos multipágina con una estructura en red (ROMERO, 2018). Siguiendo el Marco conceptual para la evaluación de la competencia lectora en PISA 2009, a continuación se presentan unas directrices que resumen las características de los diferentes tipos de textos según tres factores de clasificación: el formato y el soporte de lectura, y la tipología textual.

En cuanto al formato, hay textos continuos y discontinuos tanto en soporte impreso como digital. En este último soporte predominan, sin embargo, los textos de formato mixto y múltiple. Igualmente, la combinación de objetos no textuales con el texto tiene una presencia mucho más significativa en textos en soporte digital. Los blogs son un ejemplo de texto continuo en soporte digital. Suele tratarse de textos breves por las limitaciones de pantalla y se suele presentar una lectura fragmentada. En cambio, las páginas web creadas son generalmente textos mixtos que combinan listas, párrafos y gráficos, esquemas o audiovisuales. Los formularios en línea, mensajes de correo electrónico y foros también combinan textos continuos y discontinuos (MARCIALES; CABRA; CASTAÑEDA-PEÑA; PEÑA; MANCIPE-FLECHAS; GUALTEROS, 2012).

Otra forma de clasificar el texto es según el tipo: descriptivo, narrativo, expositivo, argumentativo e instructivo.

Sea como fuere, lo que caracteriza y define a la lectura digital (RODRÍGUEZ, 2004), y así lo contempla el PIL, tiene que ver con la personalización de la experiencia lectora, la hipertextualidad e intertextualidad, la conectividad y ubicuidad, la multimodalidad, el acceso integrado a recursos, la necesidad de competencia crítica, la interactividad y la socialización.

La personalización de la experiencia lectora tiene que ver con los objetivos, las condiciones y la naturaleza específicas de las tareas relacionadas con la lectura digital, tales como la búsqueda en Internet o el trabajo colaborativo en red, que hacen que en el diálogo entre texto y lector, el lector ocupe un lugar preeminente, en el sentido de que un mismo texto admite diferentes experiencias lectoras, porque, de hecho, el lector tiene mucho más control sobre la selección y la secuenciación de la lectura.

La hipertextualidad está relacionada con la articulación y la combinación de textos diferentes en formatos diversos mediante el enlace hipertextual, que dirigen al lector a una lectura de secuenciación selectiva mediante la navegación entre documentos diferentes. De hecho, en el hipertexto no existe una guía preestablecida de lectura y es el lector quien decide el orden. En este proceso, se establecen relaciones entre textos autónomos que generan la construcción de significados diferentes, determinados por el lector.

La conectividad entre redes diferentes hace posible el acceso a textos, documentos y fuentes de información de manera continuada y desde espacios diferentes, lo que permite una gran ubicuidad de la lectura. 
La multimodalidad y la lectura de textos en soporte digital se produce en una multiplicidad de pantallas: lector de libros digitales, móvil, ordenador, tableta, consola de videojuegos, sistemas de proyección, PDI. Esta multimodalidad incide en la capacidad de interacción del lector $\mathrm{y}$, por tanto, en una expresividad igualmente multimodal, caracterizada por la combinación de texto escrito, imagen, audiovisual, realidad aumentada, códigos QR.

El acceso integrado a la lectura digital competente se caracteriza, así mismo, por el acceso integrado a recursos de apoyo a la comprensión proporcionados por la red: diccionarios, enciclopedias, buscadores, traductores, sistemas de compartición y difusión de textos.

Consecuentemente, la necesidad de competencia crítica juega aquí un papel fundamental. A partir de la capacidad del lector para determinar la construcción de significados intertextuales, es necesaria la adquisición de habilidades específicas para el análisis crítico de lo que se lee en pantalla. Esto afecta a la búsqueda de información, la valoración de la adecuación, la autoría, la veracidad y la calidad de los textos. Una comprensión lectora competente incluye la detección de los elementos que informan de la intencionalidad y la ideología de los textos que se leen, permite eludir el exceso de información y evitar la desinformación.

La interactividad y las posibilidades de interacción de las herramientas digitales y las redes sociales permiten que el proceso lector incorpore la escritura y el intercambio de cualquier información.

Por último, la socialización tiene que ver con que el formato digital facilita la discusión en torno a diferentes aspectos de las lecturas y posibilita la colaboración entre lectores a partir del uso de las herramientas 2.0 y las redes sociales (blogs, foros, wikis, marcadores, por ejemplo).

\section{Conclusiones}

El PIL es concebido como una estrategia que integra un conjunto de directrices que para nada se quedan en un mero plano teórico. Antes bien, lo que pretende es impulsar un conjunto de acciones relacionadas con el desarrollo de la CL, que implica la comprensión, la reflexión, el discernimiento y la transferencia de conocimientos para la adaptación creativa a cada contexto. Por lo tanto, habrá que tener muy en cuenta cómo las prácticas lectoras se relacionan con las actividades que, desde las diferentes áreas curriculares y otros espacios educativos, se plantean para los aprendizajes propios de cada disciplina.

La CL, como aquí se ha explicado, es una capacidad que moviliza recursos personales, convenientemente relacionados: los conocimientos (saberes), los procedimientos (saber hacer) y, finalmente, los valores y actitudes (saber ser), que permiten implicarse en las actividades lectoras, tanto las académicas como las de la vida cotidiana (JIMÉNEZ, 2014).

Del mismo modo existe una serie de ámbitos en los que la lectura debe jugar un papel activo para la construcción del conocimiento y el desarrollo de este (MORALES; RINCÓN; TONA, 2005). Un primer ámbito que hay que atender debe hacer referencia a la amplia gama de contextos sociales y culturales -casa, escuela, mundo laboral, ocioen que es necesaria la lectura. Significará tener en cuenta tanto los usos sociales más tradicionales como los más actuales, los que se generan, por ejemplo, por la presencia de los textos que se producen con las tecnologías de la información y la comunicación. 
En segundo lugar, hay que tener en cuenta justamente cómo son los textos, en toda su diversidad (continuos y discontinuos, verbales, iconoverbales y / o multimodales, publicados electrónicamente o con otros medios y materiales de edición, con estructura hipertextual o no), para asegurar que se atienden todos los elementos que hay que considerar y para dar coherencia a las actividades de enseñanza y de aprendizaje que ayudarán a comprenderlos, a construir su significado y atribuirles valores.

Además, habrá que precisar también los recursos personales, las actitudes, las estrategias y los procedimientos que el lector o la lectora deberá utilizar en sus prácticas letradas para alcanzar objetivos personales y sociales que favorezcan su participación activa, responsable, cívica y crítica en la sociedad.

La CL supone comprender y utilizar textos de todo tipo, reflexionar e implicarse en su lectura para alcanzar los objetivos propios, desarrollar el conocimiento y todas las potencialidades personales que harán posible participar de manera consciente, crítica, responsable y cívica en una sociedad democrática. En consecuencia, tal como hemos desarrollado, para abordar adecuadamente las diferentes dimensiones de la comprensión lectora habrá que atender a los contextos sociales y culturales en que se lee, los textos y su tipología, el soporte de lectura y el lector.

La Biblioteca Escolar juega aquí un papel importante (DURBÁN, 2010). No se trata de un espacio cerrado y de almacenamiento de fondos bibliográficos de todo tipo, sino que su acceso por parte de toda la comunidad educativa es imprescindible para que el fomento de la lectura sea real y no una quimera teórica. En especial, la Biblioteca Escolar es la que debe estar provista de los fondos necesarios para adquirir una sólida competencia literaria que supere las convecciones impuestas por las lecturas literarias de tipo obligatorio.

Por último, el PIL contempla la lectura digital como una forma de lectura válida, funcional y que supone un tipo de interacción entre el texto y el lector. Es el lector el que debe contemplar todas las posibilidades de lectura que el hipertexto le ofrece. El saber contemplarlas ayuda a quien lee a formar una sólida CL digital, es decir, aquella que sabe acceder a la información hipermodal con la finalidad de aprovecharla, para así acrecentar el conocimiento de cualquier tipo. Desde este punto de vista, pantalla, texto y lector forman un trinomio potente que van más allá de la simple navegación por lenguajes digitales.

\section{Referencias}

BALLESTER, J. La formación lectora y literaria. Barcelona: Graó, 2015.

CERRILLO, P. C. Literatura Infantil y Juvenil y educación literaria. Madrid: Ediciones Octaedro S. L., 2010.

CLAR, R.; LLAURADÓ, A.; RIERA, A.; QUINQUER, D.; ROCA, M. Hablar, leer y escribir para aprender desde las áreas curriculares. Aula de Innovación Educativa, v. 159, 2007. Recuperado el 11 de mayo de 2019, de https://core.ac.uk/download/pdf/132120613.pdf

CORDÓN GARCÍA, J. A.. La investigación sobre lectura en el entorno digital. Méi: Métodos de información, v. 7, n. 13, pp, 247-268, 2016. 
DURÁN, T.; MANRESA, M. Entre països: l'acció educativa en el nostre entorn. In COLOMER, T.: (Org.). Lectures adolescents. Barcelona: Graó, 2008, p. 59-114.

DURBÁN, G. (2010). La biblioteca escolar. Un recurso estratégico para el centro. Barcelona: Graó.

GENERALITAT DE CATALUNYA. Decret 142/2007, de 26 de juny, pel qual s'estableix l'ordenació dels ensenyaments de l'educació primària. 2007. Recuperado el 11 mayo de 2019, de https://portaljuridic.gencat.cat/ca/pjur_ocults/pjur_resultats_fitxa/\%3Faction\%3Dfitxa\% 26documentId\%3D451702\%26language\%3Dca ES\%26searchId\%3D5200758\%26mod e\%3Dsingle

GENERALITAT DE CATALUNYA. L'impuls a la lectura. Una estratègia per a l'èxit escolar. 2011-2014. Recuperado el 11 de mayo de 2019, de http://xtec.gencat.cat/ca/projectes/lectura/impulslectura/

GENERALITAT DE CATALUNYA. La lectura en un centre educatiu. 2013. Recuperado el 11 de mayo de 2019, de http://ensenyament.gencat.cat/web/.content/home/departament/publicacions/monografie s/lectura/la_lectura_en_un_centre_educatiu.pdf

GENERALITAT DE CATALUNYA. Ofensiva de país a favor de l'èxit escolar. 2013. Recuperado el 11 de mayo de 2019, de http://ensenyament.gencat.cat/web/.content/home/departament/publicacions/monografie s/ofensiva-exit-escolar/ofensiva_exit_escolar.pdf

JIMÉNEZ, E. Comprensión lectora vs Competencia lectora: qué son y qué relación existe entre ellas. Investigaciones sobre lectura, v. 1, pp. 65-74, 2014.

LOMAS, C. Leer para entender y transformar el mundo. Enunciación, v. 8, n. 1, p. 5767, 2003.

MARCIALES, G. P.; CABRA, F.; CASTAÑEDA-PEÑA, H.; PEÑA BORRERO, L. F.; MANCIPE-FLECHAS, E.; GALTEROS, N. Nativos digitales. Transformaciones del formato impreso al digital. Bogotá: Pontificia Universidad Javeriana, 2013.

MARCHESI, Á; MIRET, I. Las bibliotecas escolares en España: análisis y recomendaciones. Salamanca: Fundación Germán Sánchez Ruipérez, 2005.

MORALES, Ó.; RINCÓN, Á. G.; TONA, J. Consideraciones pedagógicas para la promoción de la lectura dentro y fuera de la Escuela. Revista de Teoría y Didáctica de las Ciencias Sociales, v. 10, pp. 195-218, 2005.

MEC. Marco Pisa 2009. Programa para la Evaluación Internacional de los Alumnos. OCDE. Informe español. 2009. Recuperado el 11 de mayo de 2019, de 
https://www.mecd.gob.es/dctm/ievaluacion/internacional/pisa-2009-conescudo.pdf?documentId=0901e72b808ee4fd

RODRÍGUEZ ILLERA, J. L. Las alfabetizaciones digitales. Revista Bordón, v. 56, n. 34, p. 431-441, 2004.

ROMERO, L. Lectura tradicional versus lectura digital. Correspondencias \& análisis, v. 4, 63-75, 2014.

ROSE, D. ; MARTÍN, J. R.. Leer para aprender: lectura y escritura en las áreas del currículo. Madrid: Pirámide, 2018.

SÁEZ, T. D.; CLARIANA, M.; CLADELLAS, R.; BADIA, M.; GOTZENS, C. La lectura por placer: su incidencia en el rendimiento académico, las horas de televisión y las horas de videojuegos. Ocnos: Revista de estudios sobre lectura, v. 12, p. 107-116, 2014.

SELFA, M.; VILLANUEVA ROSA, J. D. La enseñanza de la lectura y la escritura hoy. Entrevista a Ana Teberosky. Investigaciones sobre Lectura, v. 4, p. 125-135, 2015.

SELFA, M.: BALÇA, Â.; COSTA, P.. Biblioteca escolar, lectura y literatura infantil y juvenil: selección de títulos actuales en español y portugués (2005-2015). Tejuelo, v. 25, p. 5-34, 2017.

SOLÉ, I. Leer, escribir y aprender. Aula de Innovación Educativa, v. 96, p. 6-9, 2000.

SOLÉ, I. Estrategias de lectura. Barcelona: Graó, 2011.

SOLÉ, I. Competencia lectora y aprendizaje. Revista Iberoamericana de Educación, v. 59, n. 1, p. 43-61, 2012.

SOLÉ, I. Aportaciones de la psicología a la comprensión de los procesos de lectura. Textos de didáctica de la lengua y la literatura, v. 63, p. 437-459, 2013.

Recebido em 26 de maio de 2019

Aceito em 17 de setembro de 2019 